Ann. Biol. anim. Bioch. Biophys., I964, 4 (3) 287-295.

\title{
ETUDE COMPAREE DES ACIDES GRAS RAMIFIÉS DES ALIMENTS ET DU LAIT, CHEZ LA VACHE
}

\author{
S. KUZDZAL-SAVOIE \\ Station centrale de Recherches laitières et de Technologie des Produits animaux, \\ Centre national de Recherches zootechniques, Jouy-en-Josas (Seine-et-Oise)
}

\section{SOMMAIRE}

L'expérience a porté sur un troupeau expérimental de 7 vaches laitières.

Les acides ramifiés des lipides alimentaires et du lait ont été dosés par chromatographie en phase gazeuse. On a ensuite calculé les quantités d'acides gras ramifiés ingérés et sécrétés au cours d'une période de 24 heures.

Il n'existe qu'une faible proportion d'acides gras ramifiés dans les lipides des aliments distribués aux vaches laitières (inférieure à $\mathrm{I} p$. roo des acides gras totaux).

Par contre, les acides ramifiés représentent une fraction toujours notable des acides gras du lait, voisine de 2 à 3 p. roo des acides totaux. Les acides ramifiés à I 5 et I 7 atomes de carbone sont prépondérants.

Ceci appuie l'hypothèse de l'origine microbienne, d'une partie au moins, des acides gras ramifiés du lait.

\section{I. - INTRODUCTION}

Les acides gras ramifiés se distinguent des acides à chaîne droite par la présence d'un groupe méthyle fixé sur le pénultième ou l'antépénultième carbone ou par la présence de plusieurs groupes méthyle répartis le long de la chaîne.

Ces acides sont toujours présents dans la matière grasse du lait (SHORLAND, I963; MAGIDMAN et al., I962 ; HERB et al., I962). Certains ont été isolés et identifiés : ce sont dans la série iso, les acides à I3, I4, I5, I6, I7 et I8 atomes de carbone; et dans la série anteiso les acides à I3, I5 et I7 atomes de carbone. Citons encore les acides à I9 et 20 atomes de carbone, multiramifiés (SHORLAND et HANSEN, I957; SONNEVELD et al., Ig62; HANSEN, Ig64). 
D'autres ont été seulement signalés. Ainsi, en 1956, JAmEs et al. indiquent la présence des acides à 9, IO, I I et I2 atomes de carbone et AsT et VAN DER WAL (I 96I) considèrent comme probable l'existence à l'état de traces d'un acide ramifié à 5 atomes de carbone.

Cependant les auteurs de publications récentes sur les acides gras du beurre (SHORLAND, I963 ; HERB et al., Ig62) ne citent pas d'acides ramifiés dont le nombre d'atomes de carbone soit inférieur à I3. Nous ne les avons d'ailleurs pas décelés nous-mêmes, mais cela peut être dû̀ à une sensibilité insuffisante de la méthode d'analyse utilisée.

Le total des acides ramifiés présents dans le beurre représente selon HERB et al. (I962), I,55 p. Ioo du total des acides gras. En fait le pourcentage des acides ramifiés varie d'un échantillon de beurre à l'autre. Fin général il apparaît que le taux de ces acides est plus élevé si le taux d'acide palmitique est aussi plus élevé (H.AwkE, I963).

D'après nos propres résultats, les acides ramifiés constituent le plus souvent 2 ou 3 p. Ioo des acides totaux du beurre.

On trouve également des acides ramifiés dans la matière grasse du lait d'autres espèces animales, mais en quantités toujours nettement inférieures aux quantités trouvées dans le cas des Ruminants (KuzDZAL-Savole et Kuzdzar, I963; SHORI,AND, I963).

L'origine des acides gras ramifiés du lait, n'est pas définitivement établie.

Depuis la découverte de certains acides ramifiés dans les lipides de fourrages tels que le ray-grass (SHORLAND, I96I), l'origine alimentaire des acides ramifiés du lait a été évoquée (ALIISON et al., I962).

Il nous a semblé intéressant de préciser ce point et de comparer l'importance de l'origine alimentaire vis-à-vis des autres origines possibles déjà établies, que nous exposerons ci-après.

\section{II. - MATÉRIEL ET MÉTHODES}

\section{A. - Díroulement de l'expérimentation}

En février et mars 1963 , une expérience d'alimentation était poursuivie au Centre national de Recherches zootechniques, sur un groupe de vaches laitières.

La quantité des différents aliments consommés, la quantité de lait produite et le taux butyreux étaient contrôlés.

Il nous a été possible d'utiliser quel pues vaches de ce troupeau expérimental en vue de comparer les acides gras consommés et les acides gras sécrétés dans le lait. L'ensemble de l'expérience est rapporté dans une publication récente (KUZDZAL-SAVOIE, 1964). La présente étude ne concerne que les acides ramifiés.

Au cours d'études antérieures (KuzDzal-Savole et KUzDzal, 196I), nous avions montré que la variation de composition de la matière grasse du lait provoquée par une modification au niveau du régime alimentaire apparaissait très rapidement (dès la traite suivante) et atteignait 48 heures après la modification du régime, une amplitude voisine de l'amplitude maximale.

Nous avons donc comparé les acides ramifiés des aliments consommés les jours J avec les acides ramifiés du lait produit le soir du jour $\mathrm{J}+\mathrm{I}$ et le matin du jour $\mathrm{J}+2$.

La même expérience a été effectuée à deux reprises (jours $J$ : I8 février et 28 mars) sur quatre, puis sur trois vaches. 
La ration journalière des vaches laitières du troupeau expérimental se composait de quantités variables de betteraves (I 5 à $20 \mathrm{~kg}$ ), d'ensilage (I 5 à $20 \mathrm{~kg}$ ), de foin (aggloméré, 8 à I $5 \mathrm{~kg}$, ou normal, I à $3 \mathrm{~kg}$ ) et de concentré à base de céréales (o à $5 \mathrm{~kg}$ ).

\section{B. - Déterminations analytiques}

$\mathrm{I}^{\mathrm{o}}$ Extraction des lipides el préparation des esters méthyliques.

a) $A$ partir des aliments.

Les lipides ont été extraits des différents aliments, soit directement après broyage (concentré, foin) soit après lyophilisation et broyage (betterave, ensilage).

L'extraction a été poursuivie pendant 16 heures dans un appareil d'extraction continue (B.B.S.) par un mélange d'éther de pétrole et d'alcool éthylique (90: Io, en vol.), précédemment utilisé par SHORLAND (1961).

Les extraits éthérés provenant des aliments ont été saponifiés. L'insaponifiable a été extrait, les acides gras ont été ensuite libérés par acidification et extraits à l'éther de pétrole. Après évaporation du solvant, les acides gras ont été estérifiés par l'alcool méthylique en présence d'acide sulfurique.

b) 4 partir du lait.

Les échantillons de lait ont été écrémés et lá crème a été tranformée en beurre. L'analyse porte sur la matière grasse obtenue après fusion et centrifugation du beurre.

A partir de l'huile de beurre les esters méthyliques ont été préparés par trans-estérification en présence d'hydroxyde de potassium.

\section{$2^{\circ}$ Dosage des acides ramifiés.}

Le dosage des acides ramifiés a été effectué par chromatographie en phase gazeuse effectuée sur les mélanges d'esters méthyliqucs. Un tel dosage est rendu possible par l'existence d'une différence entre les temps de rétention de l'acide ramifié et de l'acide à chaîne droite, à même nombre d'atomes de carbone. Ainsi sur colonne de polyester de Craig (succinate de butane-diol), les acides ramifiés précèdent les acides à chaîne droite (HAwKE et al., I959).

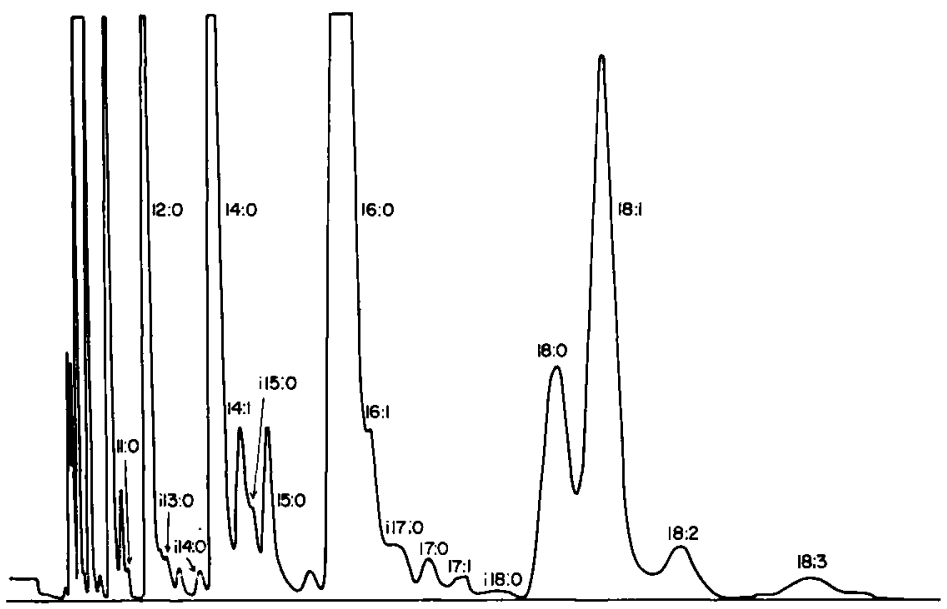

FIG. I. - Analyse par chromatographie en phase gazeuse des esters méthyliques des acides gras du beurre

Conditions de l'analyse : appareil utilisé : Aérograph (modèle 1 ro C-Wilkens, Walnut, Californie). Colonne : longueur $3,048 \mathrm{~m}$, diamètre $0,635 \mathrm{~cm}$, chargée à I 5 p. Ioo de polyester de Craig sur brique (6o-80 mesh). Température $210^{\circ} \mathrm{C}$ environ. Gaz vecteur : hélium. Débit : $50 \mathrm{ml} / \mathrm{mn}$ environ.

Les acides sont désignés par un rapport dans lequel le premier chiffre indique le nombre d'atomes de carbone et le deuxième chiffre le nombre de doubles liaisons. La lettre $i$ caractérise les acides ramifiés.

Annales de Biologie animale. - 1964 . 
Un exemple d'analyse par chromatographie en phase gazeuse des esters méthyliques des acides gras du beurre, est donné sur la figure I. On distingue nettement les pics correspondant aux acides ramifiés à $14, \mathrm{I} 5$, I 6 et I 7 atomes de carbone.

La résolution des pics correspondant d'une part à l'acide monoène à chaîne droite à $n$ atomes de carbone et d'autre part à l'acide ramifié à $n+\mathrm{I}$ atomes de carbone est imparfaite. Cependant une évaluation approximative de la surface respective des pics correspondant à ces deux types d'acide est possible. Par ailleurs, le pourcentage de l'acide monoène à 15 atomes de carbone est nettement inférieur au pourcentage de l'acide ramifié à I6 atomes de carbone (HERB et al., I962) ce qui favorise l'identification du pic correspondant à ce dernier acide. Enfin, l'acide monoène à I 3 atomes de carbone n'ayant pas été signalé dans le beurre aucun acide ne risque d'interférer avec l'acide ramifié à I4 atomes de carbone.

Nous ne différencions pas, par cette méthode, les divers types d'acides ramifiés (iso, antéeso ou multiramifié).

\section{RÉSULTATS}

Le seul aliment dans lequel nous avons décelé des acides ramifiés en quantité notable est l'ensilage. Une analyse par chromatographie en phase gazeuse des esters méthyliques des acides gras de l'ensilage est présentée sur la figure 2. On distingue

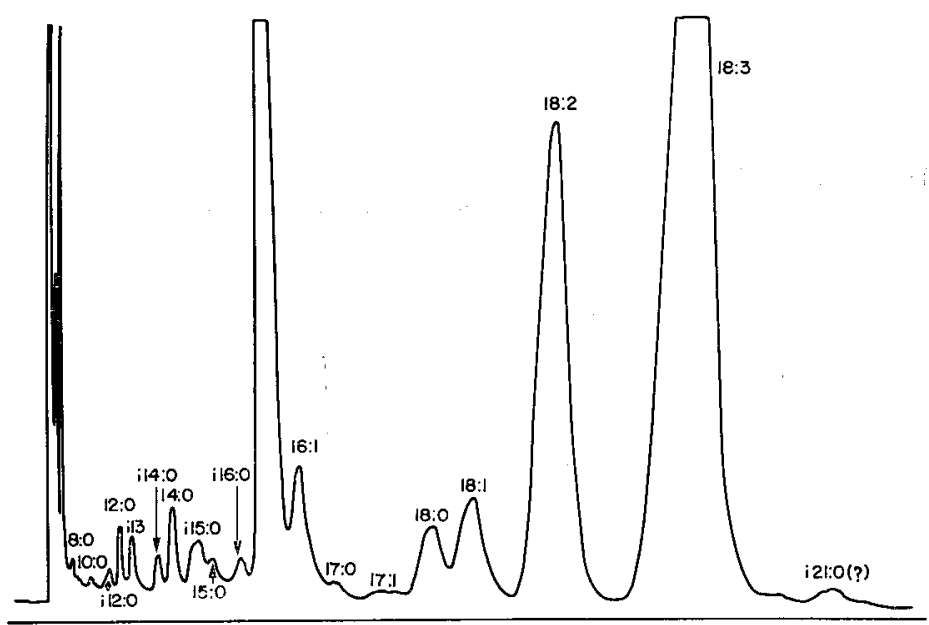

FIG. 2. - Analyse par chromalographie en phase gazeuse des acides gras de l'ensilage Conditions de l'analyse : voisines de celles indiquées sous figure I

les acides ramifiés à I2, I3, I4, I5 et I6 atomes de carbone, identifiés par la valeur du logarithme du temps de rétention à débit constant. Dans l'herbe, certains de ces acides ramifiés sont présents, mais seulement à l'état de traces (c'est-à-dire à un taux inférieur à $o, I$ p. Ioo).

Le fait que ces acides ramifiés soient relativement abondants $(2 \mathrm{p}$. Ioo environ des acides gras totaux) dans l'ensilage illustre les modifications que les fermentations microbiennes peuvent apporter au niveau des acides gras. 
La comparaison entre les acides ramifiés des aliments et les acides ramifiés du lait est présentée dans le tableau I.

\section{TABLEAU I}

Etude comparée des acides gras ramifiés consommés par la vache laitière et des acides gras ramifiés produits dans le lait

\begin{tabular}{|c|c|c|c|c|c|c|c|}
\hline & \multicolumn{3}{|c|}{ Série $1(18-2-63)$} & \multicolumn{4}{|c|}{ Série 7 (28-3-63) } \\
\hline & $\begin{array}{l}\text { Vache } \\
n^{\circ} 10.3\end{array}$ & $\begin{array}{c}\text { Vache } \\
\text { no } 9028\end{array}$ & $\begin{array}{l}\text { Vache } \\
\text { no } 158\end{array}$ & $\begin{array}{r}\text { Vache } \\
n^{0} 103\end{array}$ & $\begin{array}{c}\text { Vache } \\
\text { no } 9028\end{array}$ & $\begin{array}{r}\text { Vache } \\
n^{0} 158\end{array}$ & $\begin{array}{c}\text { Vache } \\
\text { no } 9019\end{array}$ \\
\hline \multicolumn{8}{|l|}{ Étude globale (en g) } \\
\hline Ac. gras totaux consommés $\ldots . .$. & 168,1 & $2: 31,9$ & 279,0 & 212,0 & 257,1 & 175,1 & 220,5 \\
\hline Ac. gras totaux produits dans le lait. & 463,3 & 709.3 & 517,9 & 386,3 & $56,3,2$ & 495,1 & $6 \pm 6,0$ \\
\hline Ac. gras ramifiés consommés $(\mathbf{1}) \ldots$ & 2,0 & 1,9 & 1,9 & $\mathbf{2 , 1}$ & 2,1 & 2,4 & 2,7 \\
\hline $\begin{array}{l}\text { Ac. gras ramifiés produits dans le } \\
\quad \text { lait }\left({ }^{1}\right) \ldots \ldots \ldots \ldots \ldots \ldots \ldots \ldots\end{array}$ & $1 ;+6$ & $1 \therefore, 1$ & 16,0 & 8,5 & 18,0 & 15,9 & 17,5 \\
\hline Étude détaillée $(\mathrm{en} g)\left({ }^{2}\right)$ & & & & & & & \\
\hline$i 12: 0$ & & & & & & & \\
\hline 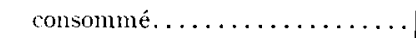 & 0,2 & $\theta$ & 0,1 & 0,2 & 0 & 0,2 & 0,2 \\
\hline${ }_{i 13: 0}^{\text {produit }} \ldots \ldots \ldots \ldots \ldots \ldots \ldots \ldots$ & 0 & 0 & 0 & 0 & 0 & 0 & 0 \\
\hline 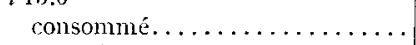 & $1), 5$ & 11,5 & $0,,^{\prime}$ & $0, \overline{5}$ & 0,6 & 0,6 & 0,7 \\
\hline produit $\ldots \ldots \ldots \ldots \ldots \ldots$ & $0,9 ?$ & $1, \mathbf{k}$ & $1,0 \div$ & $0,6 ?$ & 1,7 & 1,5 & 1,9 \\
\hline$i 1_{1}^{1}: 0 \ldots \ldots \ldots \ldots \ldots \ldots \ldots \ldots \ldots \ldots$ & & & & & & & \\
\hline consomné..$\ldots \ldots \ldots \ldots \ldots$ & 0,3 & 0,3 & 0,2 & $0, \dot{t}$ & 0,4 & 0,4 & 0,4 \\
\hline i produit $\ldots \ldots \ldots \ldots \ldots \ldots \ldots \ldots$ & $1,{ }^{\prime} t$ & $\ddot{2}, 1$ & 1,6 & 0,8 & 1,1 & 1,5 & 1,3 \\
\hline 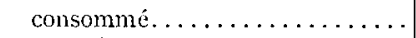 & 0,4 & $0,,^{\prime}$ & $0,,_{1}^{\prime}$ & 0,5 & 0,6 & 0,6 & 0,7 \\
\hline$i_{i 16: 0}^{\text {produit }} \ldots \ldots \ldots \ldots \ldots \ldots \ldots$ & $3,5 ?$ & $4,5 ?$ & $1,2:$ & 1,5 & 3,1 & $\iota_{t}, 0$ & 3,2 \\
\hline consommé. . . . . . . . . . & 0,5 & $0, \bar{j}$ & $0,,^{\prime}$ & 0,5 & 0,6 & 0,6 & 0,7 \\
\hline produit $\ldots \ldots \ldots \ldots \ldots \ldots$ & 2,3 & 2,8 & 3,1 & $1,3 ?$ & 1,7 & $1,5 ?$ & 1,4 ? \\
\hline i $17: 0$ & & & & & & & \\
\hline consommé $\ldots \ldots \ldots \ldots \ldots \ldots$ & 0 & 0 & 0 & 0 & 0 & 0 & 0 \\
\hline $\begin{array}{l}\text { produit } \ldots \ldots \ldots \ldots \ldots \ldots \ldots \ldots \\
i \text { 18:0 }\end{array}$ & 5,1 & 5,7 & 5,2 & 3,9 & 7,3 & 5,9 & 7,8 \\
\hline consommé $\ldots \ldots \ldots \ldots \ldots \ldots$ & 0,1 & 0,2 & $0,,_{t}^{\prime}$ & 0 & 0,2 & 0 & 0 \\
\hline produit .............. & $1, x^{\prime}$ & $1, i ?$ & $1,5 ?$ & 0,4 & 2,8 & 1,5 & 1,9 \\
\hline
\end{tabular}

(1) Total approximatif.

(2) $i$ signifie acide ramifié. Le ${ }^{\text {er }}$ chiffre du rapport est le nombre d'atomes de carbone ; le $2^{e}$ chiffre du rapport indique le nombre de doubles liaisons (ici tous les acides sont saturés).

L,es quantités totales d'acides ramifiés ont été calculées. Ces résultats sont parfois approximatifs mais la présence de sept bilans concordants, donne à l'ensemble une réelle solidité.

Ajoutons également que les quantités d'acides gras ramifiés présentées dans le tableau $\mathrm{I}$, s'alignent sur les résultats récents publiés par divers auteurs (HERB et al., I962; HaWkE, Ig63; KaUfManN et MANKeI, I963).

Annales de lsiologie animale. - 1964 . 
L'étude du tableau I nous permet de faire plusieurs observations.

Tout d'abord la quantité d'acides gras ramifiés sécrétée dans le lait est nettement supérieure à la quantité ingérée.

Ensuite, on observe une certaine proportionnalité entre les quantités respectives d'acides gras ramifiés et d'acides gras totaux sécrétés dans le lait: le rapport des deux quantités varie seulement de 2,2 à 3,2 pour les différentes vaches du troupeau expérimental alors que les taux extrêmes d'acides ramifiés varient du simple à plus du double.

La vache Io3 qui sécrète le moins d'acides ramifiés est aussi celle qui sécrète le moins d'acides gras totaux.

Enfin une dernière observation découle de l'étude détaillée des différents acides ramifiés (tabl. I) qui the se comportent pas tous da la même manière.

Ainsi l'acide ramifié en $\mathrm{C}_{12}$ n'est pas décelé dans le lait. La différence entre les acides ramifiés ingérés et sécrétés est moins grande pour les acides à $\mathrm{I} 4$, I 6 et I 8 atomes de carbone que pour les acides à $\mathrm{I} 5$ et $\mathrm{I} 7$ atomes de carbone (ces derniers représentent la fraction principale des acides ramifiés du lait).

Nous n'avons pas décelé par la technique d'analyse utilisée d'acide $i$ so- $\mathrm{C}_{17}$ dans la ration. SHORLAND (I96I) signale cependant l'existence de cet acide dans le ray-grass à raison de 0,2 p. Ioo des acides totaux. Admettant ce chiffre, la quantité ingérée serait cependant loin d'atteindre la quantité sécrétée : 5 à $9 \mathrm{~g}$ en 24 heures.

Il apparaît ainsi, sans aucune ambiguïté, que l'origine alimentaire des acides gras ramifiés — si elle existe - ne pourrait expliquer que la présence d'une fraction minime des acides ramifiés tronvés dans le lait.

Cette assurance, qu'il nous semblait nécessaire d'acquérir en premier lieu, nous permet maintenant de discuter des autres origines possibles des acides ramifiés présents dans le lait.

\section{IV. - DISCUSSION}

SHORLAND (I963) a rappelé récemment la suggestion formulée en I952 par EL, SHazLY, selon laquelle les acides gras volatils ramifiés (iso-butyrique, iso-valérique ou 2-méthyl butyrique) provenant de la dégradation des acides aminés et trouvés dans le rumen (ANNISON, I954), pouvaient conduire, par un mécanisme de biosynthèse, aux acides gras ramifiés à haut poids moléculaire.

Une telle synthèse serait possible in vitro (HoRnING, I96I) mais in vivo elle ne semble pas avoir été définitivement démontrée (VERBEKE et al., I959; BHALERAO, I96I). Les acides ramifiés présents dans la matière grasse du lait peuvent d'ailleurs avoir une autre origine.

Rappelons que le pourcentage des acides ramifiés dans le lait des Ruminants est toujours supérieur au pourcentage de ces mêmes acides dans le lait d'autres espèces animales (SHORLAND, I963). Or les lipides du protoplasme des microorganismes contiennent une quantité importante d'acides ramifiés, iso- $\mathrm{C}_{15}$ et $i$ so- $\mathrm{C}_{17}$ en particulier (SAITO, I960; AliISON et al., I962; KEENEY et al., I962). KEENEY et al. estiment qu'environ la moitié de l'acide iso- $\mathrm{C}_{\mathbf{1 5}}$ du lait de vache peut avoir une origine microbienne. Les acides gras ramifiés provenant des micro-organismes du rumen 
sont métabolisés et suivent le système circulatoire. L,es analyses de KEENEY et al. (I962) montrent qu'ils existent a11 niveau des différents constituants lipidiques du sérum sanguin de bovin (glycérides et acides libres, esters de cholestérol et phospholipides).

On peut admettre que les acides ramifiés parviennent à la glande mammaire sous une forme ou une autre et qu'ils y sont libérés et constituent avee les autres acides gras, le " pool " d'acides gras qui existe vraisemblablement à ce niveau, pour être ensuite réincorporés dans les glycérides et les phospolipides du lait où leur présence a été démontrée. (LuIck et LuCas, I962; PATTTON et CARThy, I963; Badings, I962; MATTSSON, Ig62; SMITH et LOWRY, Ig62).

Cette origine microbienne des acides gras ramifiés du lait de vache est également admise par GARTON (I963) et par KAy et HoBson (I963), dans deux études récentes se rapportant à l'origine des acides gras du lait.

Les acides ramifiés pourraient aussi provenir des graisses de réserve. Les acides ramifiés (principalement les acides à $\mathrm{I}_{5}$ et $\mathrm{I}_{7}$ atomes de carbone) ont été étudiés et dosés récemment dans les graisses de réserves des ruminants (SzONYI, I962 ; SHORLAND, I I63) et dans celles des non-ruminants où ils existent en moindre quantité (SHorland, I963). Peut-être, les graisses de réserves fournissent-elles dans la période de forte production du lait, une certaine quantité d'acides ramifiés qui pourrait contribuer à maintenir un taux relativement constant d'acides ramifiés dans le lait?

Reçu pour publication en mai 1964.

\section{SUMMARY}

COMPARATIVE STUDY OF BRANCHED FATTY ACIDS IN THE LIPIDS OF COW'S MILK AND THE FEED

Seven milking cows were used in the experiment.

Gas-liquid chromatography was used for the determination of the branched fatty acids present in the lipids of both the feed and milk.

The amount of branched fatty acids ingested and secreted during twenty-four hours were then calculated.

The branched fatty acids in the feed lipids is only i per cent of the total fatty acids.

On the other hand, the branched fatty acids in milk form approximately two to three per cent of the total fatty acids. The major part of these branched fatty acids have fifteen and seventeen carbon atoms.

This corroborates the hypothesis that they are of microbial origin, at least in part.

\section{RÉFÉRENCES BIBLIOGRAPHIQUES}

Allison M. J., Bryant M. P., Katz I., Keeney M., ig62. Metabolic function of branched-chain volatile fatty acids, growth factors for Ruminococci. II. Biosynthesis of higher branched-chain fatty acids and aldehydes. J. Bact., 83, ro84-ro93.

Annison E. F., 1954. Some observations on volatile fatty acids in the sheep's rumen. Biochem. J., 57, 400-404. 
Ast ${ }^{-}$II. J., VAN deR WaL R. J., I961. The structural components of milk triglycerides. J. Amer. Oil Chem. Soc., 38, 6;-69.

Badings H. T., I 962 . Fatty acid composition of cow's milk phospholipids as determined by gas chromatography and thin-layer chromatography. Neth. Milk Dairy J., 16, 217-225.

Bhalerao V. R., Endores J., Kummerow F. A., ig6i. Fatty acid composition of lipids extracted from rats fed milk fat, corn oil and lard. J. Dairy Sci., 44, 1 283-1292.

El Shazly K., 1952. Degradation of protein in the rumen of the sheep. 2. The action of rumen microorganisms on amino-acids. Biochem. J., 51, 647-656.

Garton G. A., 1963. The composition and biosynthesis of milk lipids. J. Lipid Res., 4, 237-254.

Hansen R. P., I964. 2,6,10,14-tetramethylpentadecanoic acid, a constituent of butterfat. Nature (Lond.), 201, 192.

HAWkE J. C., I963. Studies on the properties of New Zealand butterfat. VIII. The fatty acid composition of the milk fat of cows grazing on rye-grass at two stages of maturity and the composition of the rye-grass lipids. J. Dairy Res., 30, 67-75.

Hawke J. C., Hansen R. P., Shorland F. B., 1959. Gas liquid chromatography : retention volumes of the methyl esters of fatty acids with special reference to $n$-odd-numbered, iso and $(+)$-anteisoacids. J.Chromat., 2, 547-55 I.

Herb S. F., Magidman P., Luddy F. Ti., Riemenschneider R. W., i962. Fatty acids of cow's milk. B. Composition by gas-liquid chromatography aided by other methods of fractionation. J. Amer. Oil Chem. Soc., 39, I 42-I 46.

IIORning II. G., Martin D. B., Karmen A., VAGElos J., 196r. Fatty acid synthesis in adipose tissue. II. Enzymatic synthesis of branched-chain and odd-numbered fatty acids. J. Biol. Chem., 236, 669-672.

James A. T., Peeters G., Lauryssens M., 1956. The metabolism of propionic acid. Biochem. J., 64, $726-730$.

Kaufmann H. P., Mankel G., 1963. Die zusammensetzung des Kuhmilchfettes in Abhängigkeit von der Fütterung. Fette Seifen Anstrich., 65, 295-302.

Kay R. N. B., Hobson P N, I963. Reviews of the progress of dairy science. Section A. Physiology. I. Physiology of the rumen. 2. Rumen microbiology. J. Dairy Res., 30, 26r-3r3.

KEeney M., Katz I., Allison M. J., ig62. On the probable origin of some milk fat acids in rumen microbial lipids. J. Amer. Oil Chem. Soc., 39, I98-201.

Kuzdzal-Savoie S., Kuzdzal W., 196r. Influence de la mise à l'herbe des vaches laitières sur les indices de la matière grasse du beurre et sur les teneurs en différents acides gras polyinsaturés. Ann. Biol. anim. Bioch. Biophys., 1, 47-69.

KuzDzAL-Savoie S., i 964 . Influence de la composition de la ration alimentaire sur la composition chimique du beurre de vache. Thèse, Faculté des Sciences, Paris, 20 avril Ig64 (sous presse).

Kuzdzal-Savoie S., Kuzdzal W., 1963. Contribution à l'étude des acides gras du lait de différentes espèces animales. Le Lait, 43, 369-384.

Luick J. R., Lucas J. M., I962. Further studies on milk fat synthesis. Proc. Soc. Exp. Biol. Med., 110, $275^{-277}$.

Magidman P., Herb S. F., Barford R. A., Riemenschnelder R. W., 1962. Fatty acids of cow's milk. A. Techniques employed in supplementing gas liquid-chromatography for identification of fatty acids. J. Amer. Oil Chem. Soc, 39, I $37^{-1} 4^{2}$.

Mattsson S., I962. Fatty acids in milk phospholipids. XVIth Intern. Dairy Cong., A (II), 537-542.

Patton S., McCarthy R. D., I963. Structure and synthesis of milk fat. IV. Role of the mammary gland with special reference to the cholesterol esters. J. Dairy Sci., 46, 396-400.

SAITo K., 1960. Studies on bacterial fatty acids. Structure of subtilopentadecanoic and subtiloheptadecanoic acids. J. Biochem. (Japon), 47, 7 10-719.

SHorland F. B., rg6r. Acetone soluble lipids of grasses and other forage plants. II. General observation on the properties of the lipids with special reference to the yield of fatty acids. J. Sci. Food Agric., 12, $39^{-42}$.

Shorland F. B., I963. The fatty acids of the depot and milk fats of Ruminants. Fette Seifen Anstrich., 65, $302-306$.

Shorland F. B. et Hansen R. P., 1957. The minor fatty acid constituents of butterfat. A review. Dairy Sci. Abstr., 19, 168-189.

Smith L. M. et Lowry R. R., 1962. Fatty acid composition of the phospholipids and other lipids in milk. J. Dairy Sci., 45, 58I-588.

Sonneveld W., Haverkamp Begemann P., Van Beers C. J., Keuning R., Shogt J. C. M., ig62. 3,7,1 1, I5-Tetramethylhexadecanoic acid, a constituent of butterfat. J. Lipid Res., 3, 35 I-353.

Szonyi C., CRASke J.D., TAIT R. S. et Pouzos A., ig62. The determination of mutton tallow in mutton! beef tallow mixtures. J. Amer. Oil Chem. Soc., 39, I85-188.'

Verbeke R., LAURYSSENS M., PeEters G., JAMES A.T., I959. Incorporation of DL-(I-14C) Leucine and ${ }_{\left.\mathrm{I}^{-14} \mathrm{C}\right)}$ iso-valeric acid into milk constituents by the perfused cow's udder. Biochem. J., 73, 24-29. 